\title{
Unexpected Spreading of Severe Allergic Reaction to Mupirocin Ointment around a Chemical Burn Wound: A Rare Case Report
}

\author{
Du-Heon Seo ${ }^{1,2}$ (D), Jin Yong Shin ${ }^{1,2}$ (D), Si-Gyun Roh ${ }^{1,2}$ (D), Suk Choo Chang ${ }^{1,2}$ (D), Nae-Ho Lee Le $^{1,2}$ \\ ${ }^{1}$ Department of Plastic and Reconstructive Surgery, Jeonbuk National University Medical School, Jeonju; ${ }^{2}$ Research Institute of Clinical Medicine of Jeonbuk \\ National University-Biomedical Research Institute of Jeonbuk National University Hospital, Jeonju, Korea
}

\begin{abstract}
Mupirocin ointment is a topical antibiotic approved and used for the treatment of cutaneous infections. Despite some side effects, mupirocin has been reported to be safe and to cause allergic reactions in only extremely rare cases. A 41-year-old male patient visited our hospital after suffering a chemical burn on his left elbow. Mupirocin ointment and a foam dressing were applied daily. After the mupirocin treatment, an exfoliative skin lesion and bullae appeared and quickly spread to the entire left upper extremity. Our clinical diagnosis of this rare case was severe allergic contact dermatitis caused by the mupirocin ointment. Application of mupirocin was terminated, and the spread of aggravated erosive cutaneous lesion was treated with normal saline and wet-gauze dressing, after which most of the erosive lesion started to heal. After 2 more weeks, there was full epithelization and the wound healed completely. This paper is a report on a rare case of severe allergic contact dermatitis resulting from treatment with mupirocin ointment.
\end{abstract}

Keywords: Mupirocin; Contact dermatitis; Allergic reaction; Burn, chemical; Wound aggravation

\section{Introduction}

Mupirocin ointment is a topical antibiotic approved for the treatment of cutaneous staphylococcal and streptococcal infections [1]. Despite its side effects, which include mild burning sensations and pain, mupirocin has been reported to be safe and well tolerated and is known to cause allergies in only extremely rare cases. To date, a small number of cases of mupirocin-induced contact allergic dermatitis have been noted [1-3]. We report a rare case of severe allergic contact dermatitis resulting from treatment with mupirocin ointment. Written informed consent to publish case details was obtained from the patient.

\section{Case}

A 41-year-old male patient without any allergic history visited our hospital after sustaining a chemical burn on his left elbow from contact with an unknown acid. At that time, the wound was limited to the elbow area, was $4 \times 4 \mathrm{~cm}$ in size, and appeared to be an erythematous lesion with minimal ruptured bullae (Fig. 1). To prevent wound infection and to provide moisture, mupirocin ointment (Esroban Oint; JW Shinyak, Seoul, Korea) and a foam dressing (MediTouch; Ildong Pharmaceutical Co., Ltd., Seoul, Korea) were applied daily while for parts of the wound with first-degree chemical burns, only the mupirocin ointment was used.
Case Report

Received: June 8, 2020

Revised: July 8, 2020

Accepted: July 9, 2020

\section{Corresponding author:}

Jin Yong Shin, M.D.

Department of Plastic and Reconstructive Surgery, Jeonbuk National University Hospital, 20 Geonji-ro, Deokjin-gu, Jeonju 54907, Korea Tel: +82-63-250-1860

Fax: +82-63-250-1866

E-mail: psjyshin@gmail.com

This is an Open Access article distributed under the terms of the Creative Commons Attribution Non-Commercial License (https://creativecommons.org/licenses/by-nc/4.0/) which permits unrestricted non-commercial use, distribution, and reproduction in any medium, provided the original work is properly cited.

C) 2020 Korean Wound Management Society 

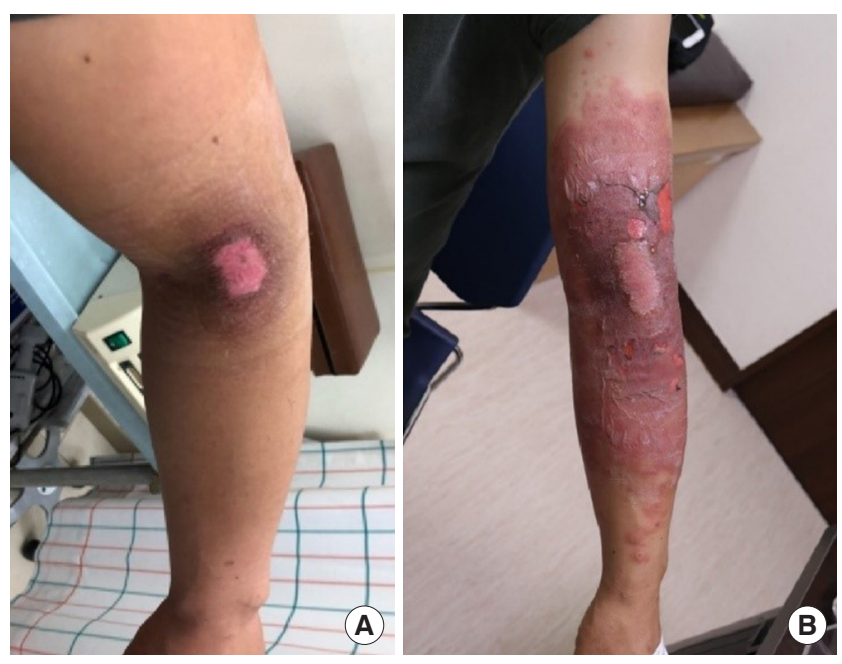

Fig. 1. Spreading wound aggravation after initial treatments. (A) The initial wound was observed to be limited to the elbow area, with erythematous lesion and minimal ruptured bullae. (B) After mupirocin ointment was applied, an exfoliative skin lesion and bullae formation appeared and quickly spread to the entire left upper extremity beyond the initial wound area.

After 2 days of treatment, the patient experienced an itching sensation, and a cutaneous erosive lesion gradually spread around the initial wound. As the wound exacerbation was considered a result of aggravation of the chemical burn, the method of dressing was maintained under observation. However, on the 3rd day of treatment an exfoliative skin lesion and bullae formation appeared where the mupirocin ointment had been applied, and this lesion quickly spread to the entire left upper extremity (Fig. 1). Because the patient had a history of several treatments with same foam materials in simple wounds, and also because the allergic reaction spread beyond the area where foam materials had been applied, the diagnosis was a severe allergic contact dermatitis to mupirocin ointment rather than an allergic reaction to foam materials. The use of mupirocin was terminated, and the spreading and aggravated erosive cutaneous lesion was treated with normal saline and wet-gauze dressing. Additionally, to subside the itching sensation during treatment, an antihistamine was prescribed. In consideration of the adverse effects of topical or oral steroids such as delayed re-epithelization and wound healing, no steroid cream was used. After the use of mupirocin was discontinued, there was notable improvement of pruritis and reduced expansion of the wound.

One week after changing the dressing method, most of the erosive lesion had started to re-epithelize, and after 2 more weeks, there was full epithelization and the wound had com-
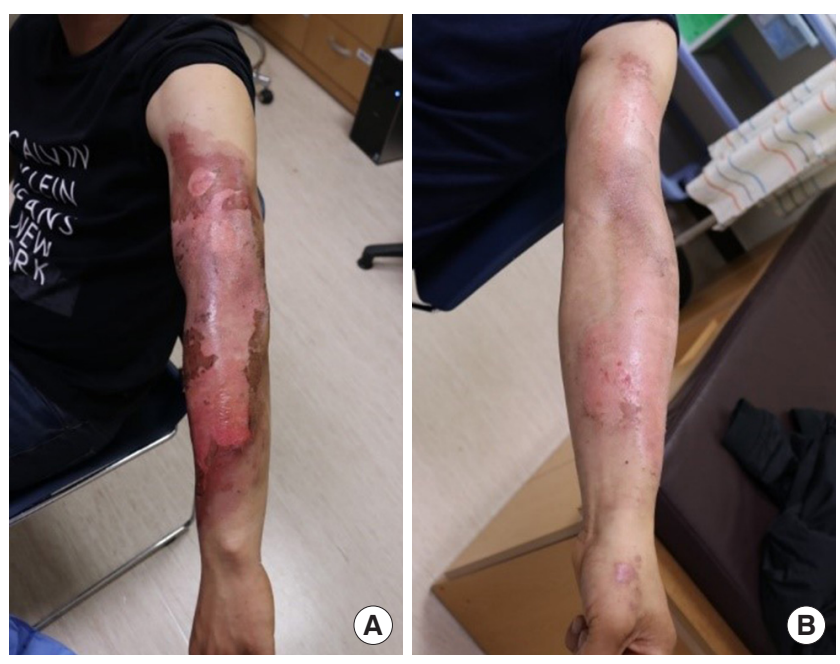

Fig. 2. Healed wound after modification of the dressing method. (A) Re-epithelized lesions were observed on the left upper extremity after use of mupirocin had been terminated and the dressing method had been modified. (B) After another 2 weeks, there was full epithelization and the wound had completely healed.

pletely healed (Fig. 2). Our clinical diagnosis of this rare case was severe allergic contact dermatitis caused by mupirocin ointment.

\section{Discussion}

Mupirocin ointment is used as a topical antibiotic worldwide to treat or prevent staphylococcal and streptococcal infections. Its active ingredient was extracted from Pseudomonas fluorescens in $1971[1,4]$. Mupirocin reversibly binds to bacterial isoleucyl-transfer RNA synthetase and thereby suppresses bacterial protein and RNA production in cutaneous infectious lesions [5].

Because mupirocin is effective for treating most cases of cutaneous infection, it has been used in a variety of wounds. This drug is particularly useful for treating both primary and secondary superficial skin infections caused by Staphylococcus aureus isolates (such as furuncle, impetigo, surgical wounds), usually with $80 \%$ improvement in infected patients and $90 \%$ eradication in the $S$. aureus isolates. Furthermore, mupirocin ointment is applied to wounds associated with cutaneous defects to prevent cutaneous infections and to provide a moist, sterile environment that facilitates optimal healing [6].

In most cases, when this ointment is applied to wounds, the only side effects and cutaneous symptoms are a temporary burning sensation or pain, observed in less than $3 \%$ of pa- 
tients. With only rare reports of contact allergic reaction to mupirocin, there were no known cases of systemic side effects that were severe enough to evoke anaphylaxis or diarrhea [7].

In our case, because the elbow wound was caused by a chemical burn from an unknown acid, we expected that it might exacerbate but only in the initial wound area. However, after applying mupirocin ointment to the wound, aggravated cutaneous symptoms, including bullae formation, skin exfoliation and pruritis, were observed over the treated area. Initially, these intensified symptoms were regarded as a manifestation of the natural course of the chemical burn instead of a rare allergic reaction to mupirocin, and as a result, lesions severely worsened and spread to the entire left upper extremity.

As mentioned above, very few cases of mupirocin contact allergic dermatitis or reaction have been reported [1-3], and no case of severe allergic reaction to mupirocin has been described. Because such cases are largely unheard of, surgeons or physicians unaware of the association between mupirocin and allergic reactions are likely to be confounded when they encounter such a case. We hope our report will raise awareness on this rare situation and enable proper decision-making to prevent exacerbation or spread of the wound.

In conclusion, allergic reaction or dermatitis resulting from mupirocin ointment is rarely reported. When a surgeon or physician encounters a situation similar to this rare case, hopefully our report will help them make correct diagnoses and decisions to prevent the wound from worsening or spreading.

\section{Conflict of interest}

No potential conflict of interest relevant to this article was reported.

\section{ORCID iDs}

Du-Heon Seo

https://orcid.org/0000-0002-0152-3191

Jin Yong Shin

https://orcid.org/0000-0003-2680-7411

Si-Gyun Roh

https://orcid.org/0000-0003-2865-0075

Suk Choo Chang

Nae-Ho Lee

https://orcid.org/0000-0003-0679-182X

https://orcid.org/0000-0003-1354-8203

\section{References}

1. Assier H, Hirsch G, Wolkenstein P, et al. Severe contact allergy to mupirocin in a polysensitized patient. Contact Dermatitis 2019;80:397-8.

2. Zhang AJ, Warshaw EM. Allergic contact dermatitis caused by mupirocin and pimecrolimus. Contact Dermatitis 2019; 80:132-3.

3. Zappi EG, Brancaccio RR. Allergic contact dermatitis from mupirocin ointment. J Am Acad Dermatol 1997;36(2 Pt 1): 266.

4. Khoshnood S, Heidary M, Asadi A, et al. A review on mechanism of action, resistance, synergism, and clinical implications of mupirocin against Staphylococcus aureus. Biomed Pharmacother 2019;109:1809-18.

5. Thomas CM, Hothersall J, Willis CL, et al. Resistance to and synthesis of the antibiotic mupirocin. Nat Rev Microbiol 2010;8:281-9.

6. Dutta P, Das S. Mammalian antimicrobial peptides: promising therapeutic targets against infection and chronic inflammation. Curr Top Med Chem 2016;16:99-129.

7. Ward A, Campoli-Richards DM. Mupirocin: a review of its antibacterial activity, pharmacokinetic properties and therapeutic use. Drugs 1986;32:425-44. 